\title{
Cardiomyopathy protection in Chagas Disease
}

\author{
Jorge E. Mitelman ${ }^{1,2,3}$, Luisa Gimenez ${ }^{4,5,6,7}$ \\ ${ }^{1}$ Instituto Centenario, Buenos Aires, Argentine \\ ${ }^{2}$ Chagas Disease of the Inter-American Society of Cardiology, Buenos Aires, Argentine \\ ${ }^{3}$ Department of Chagas, the University Institute of Health Science Foundation H A Barcelo, Buenos Aires, Argentine \\ ${ }^{4}$ Teodoro Alvarez Hospital, Buenos Aires, Argentine \\ ${ }^{5}$ Cardiology in Northern Province of Buenos Aires, Buenos Aires, Argentine \\ ${ }^{6}$ Chagas in the City of Buenos Aires, Buenos Aires, Argentine \\ ${ }^{7}$ University Institute of Health Science Foundation H A Barcelo, Buenos Aires, Argentine \\ Email: jorgemitelman@yahoo.com.ar
}

Received 28 July 2013; revised 29 August 2013; accepted 15 September 2013

Copyright (c) 2013 Jorge E. Mitelman, Luisa Gimenez. This is an open access article distributed under the Creative Commons Attribution License, which permits unrestricted use, distribution, and reproduction in any medium, provided the original work is properly cited.

\begin{abstract}
There is some published evidence suggesting micro vascular endothelial dysfunction and dysautonomia involvement in Chagas disease in association with cardiomyocyte changes favoring disease progression. The combined treatment between angiotensin converting enzyme (ACE) inhibitor drugs; Simvastatin, muscarinic antibody immunoadsorbent together with fungicidal drugs would open therapeutic possibilities in this disease.
\end{abstract}

Keywords: Chagas Disease; Chronic Period without Demonstrable Pathology; Ex Indeterminate Phase; Endothelial Dysfunction; Cardiac Dysautonomia; Simvastatin; ACE (Angiotensin Converting Enzyme) Inhibitor; Muscarinic Antibody

\section{INTRODUCTION}

Chagas Disease, American trypanosomiasis, is a parasitic disease coming from rural environments and caused by the flagellated protozoan, Trypanosoma cruzi. Chagas disease is endemic throughout much of Mexico, Central America, and South America where the estimated 8 million people are infected, but in the last few years, it has been observed in the United States of America, Canada, Europe and including Japan. This is the product of internal and external migrations of people between Latin America and the rest of the world [1-4]. The Trypanosoma cruzi parasites are transmitted by the infected faeces of blood-sucking triatomine bugs. These bugs live in adobe houses and thatched roofs in rural and suburban areas. Generally they become active at night when they feed on human blood. They usually bite an exposed area of skin such as the face, and the bug defecates close to the bite. The parasites enter the body when the person instinctively smears the bug faeces into the bite. Other forms of transmission are through blood transfusions, congenital transmission (passing from mother to baby), organ donations and recently by oral transmission of contaminated juices. Chagas disease is recognized as one of the most unattended tropical diseases as a disease of negligence and poverty and continues to be a relevant social and economic concern in the majority of the countries of Latin America. The medical and social impact of Chagas disease is high. For instance, it is estimated that about 752,000 of working years per year are lost due premature deaths caused by disease in the American countries [5].

Chagas disease goes through two stages, one is acute and the other chronic. During the acute stage, a large number of parasites circulate in the blood stream and symptoms can include fever, lymphaldenopathy, the enlargement of the liver and spleen and occasionally, myocarditis or meningoencephalitis with a serious prognosis. In some cases, the Romana's sign (palpebral edema) is present. During this period, treatment with parasiticides, like benznidazole or nifurtimox, is successful. In the chronic stage, the parasites are present; however, they are not easy to find. Of all the chagasic patients that arrive and are attended: between 30 to 40 per cent tend to have serious cardiomyopathy (arrhythmias and heart failure) or pathologic dilation (megaviscera) of the esophagus and colon. The importance of trypanosomiasis is particularly given because of its high prevalence, the large economic losses due to the inability to work and sudden death as well as the impact on public health due to high costs for prolonged hospitalization, 
medicine, heart transplants, and the implant of electronic devices like pacemakers, cardiac defibrillators and resynchronization devices.

Unfortunately, in spite of the time that has passed since Carlos Chagas discovered this nosology, up until now and even today, there are still doubts regarding the mechanisms that determine the unfavorable evolution in some of the sick. Hypotheses have been postulated that link the infected strains whether these are myotropic or pantropic, with neurological, immunological or vascular interpretations, etc.

\section{PATHOPHYSIOLOGY}

Intervening factors in the development of cardiomyopathy [6-10].

1) Intrensic myocardial damage during the acute and chronic phase because of:

a) Direct parasitic action.

b) Immunological disturbances.

c) Neurological damage.

2) Induced disorders due to the host immune response.

3) Alterations in the automatic nervous system.

4) Microvascular lesions with alterations in the microcirculation and subsequent myocytolysis.

Based on these findings, we can confirm that significant efforts have been made to prevent the infection; however, initiatives have not been developed to prevent the manifestations of cardiomyopathy. During the chronic period, the lack of resources for antiparasitic and therapeutic drugs has been confused with the impossibility to act with broader medical and therapeutic criteria than those that are exclusively antiparasitic.

In the evolutionary characterization of the so called Chagas disease, a wide range of chronic affected patients has been found without evidence of demonstrable pathology with the current diagnostic methods. This would not allow them to be considered sick. It is important to highlight the evidence that denies the fact that during this chronic period (without evidence) nothing happens. On the contrary, dysautonomy has been detected early, alterations in the microvasculature, in the diastolic function as well as the presence of antibodies against recaptors to neurotransmitters in a significant percentage and sudden death. Therefore, today the idea that the simple presence of reactive serology must be considered as a risk factor for the development of cardiomyopathy and sudden death and that this could occur in a later period, forces us to consider that this risk must be prevented by configuring protocols that are inclined to avoid the remodeling, to improve the dysfunction and to homogenize the refractory periods (O. Mordini) $[11,12]$.

\section{DIAGNOSIS OF CHAGAS DISEASE}

The diagnosis is based on three fundamental parameters:
1) epidemiological history (having been born or having lived in an endemic area), 2) laboratory (reactions from specific laboratories) and 3) clinic.

The laboratory techniques used in acute Chagas are the direct search for the parasite in the blood. The firstline test to confirm the diagnosis of acute-phase disease is direct examination of blood, by microscopy of fresh preparations of anticoagulated blood, in the chronic phase it can be confirmed by blood; xenodiagnosis; and PCR. Positive PCR results can occur in chronic infection in the absence of reactivation, and also it can be detected through immunoglobulins in an indirect form. Today they are: Indirect immunoflurescence (IFA); EnzymeLinked Immunosorbant Assay (ELISA); Indirect Hemogglutination test (IHA); the Particle Agglutination test (PA), requiring the positivization of two techniques. Therefore, if one is positive and the other is negative then a third study must be carried out.

Every patient with positive serology for Chagas disease must be incorporated in the secondary prevention. On March 20, 2010, in the Four Seasons Hotel of Buenos Aires, it was agreed that the State would guarantee the access to the following diagnostic exams for every patient with Chagas disease. Routine laboratory tests of: the complete blood count (hemogram), glycemia, urea, creatinemia, electrolytes, cholesterol, aminotransferase, liver, urinalysis and a chest X-ray.

Electrocardiogram with twelve derivations.

Gradual cardiac stress test according to the Bruce protocol.

Two-dimensional echocardiography, doppler echocardiogram and tissue doppler imaging.

Holter for 24 hours.

Measurement of antibodies against muscarinic recaptors.

Rest/stress gated 99 m Tc-sestamibi myocardial perfusion SPECT to evaluate simultaneously the perfusion and function. Radioisotope ventriculography to evalutate the systolic function and gadolinium enhanced Cardiac Magnetic Resonance to evaluate the presence or not of myocarditis.

Radiologic studies contrasted with the gastrointestinal transit [11,12].

Diverse clinical studies would suggest an initial extensive vasculoendothelial compromise, detected in some patients in the chronic phase without proven ex indeterminate pathology and more frequent in the chronic phase with cardiopathy, causing profound changes in the myocardial cells. The parasite could harm the endothelium but it is not truly known how this is done [13,14].

Some authors have postulated that the action of the nueraminidase (of the parasite) would act by removing the electronegative groups of sialic acid of the phosphosphingolipids from the membrane (which normally 
facilitate the blood flow by electrostatic rejection) increasing the resistance to the flow and altering the normal state of the microcirculation [9]. This damage or disturbance of the endothelium, secondary to the loss of sialic acid not only would complicate the microcirculation but would also predispose to thrombosis, by increasing viscosity and platelet aggregation.

With regard to the last position, Jorge and his collaborators in 1974 and more recently with views from Tanowitz focused their attention on the microvascular endothelial lesion.

Other authors have reported that the endothelium would respond to the action of the inflammatory cytokines, altering the production of nitric oxide (NO), which could have a connection with the chagasic pathogenesis.

Whatever the original mechanism may be, provoked alterations in the endothelial function would elevate the platelet reactivity and the platelet-endothelial cell interaction producing modifications in the structured proteins, biochemical disturbances in the signal transduction with changes in the proliferation, cell function and inflammatory responses. Once the lesion is established in the microcirculation, the ischemia would produce myocytolysis replacing the cardiac tissue with fibrosis. The platelet aggregation, the microthrombosis and the contact between lymphocytes and endothelial cells would contribute to the progression of the illness. The von Willebrand factor is a protein produced by the endothelial cell and the megakaryocytes. It is present in the plasma, platelets and vascular endothelium forming unions between the collagen of the subendothelium and the glycolproteins of the platelets. The endothelium, in presence of functional alterations like those described beforehand, puts in risk the mechanisms of the thrombus resistance, intervening among other factors that of Von Willebrand, resulting in a marker of endothelial disturbance that allows for its increase and the early detection of this phenomenon [15-17].

The abnormalities of the coronary microvascular and epicardial vasomotion due to the dysfunction of the vascular endothelium was described among other areas by our group with a brachial echo Doppler and the von Willebrand factor; from Dr. Harry Aquatella through the realization of the acetylcholine test given to chagasic patients in different stages and from Dr. Perez Baliño with gamma camera [14].

All this evidence would suggest that the myocardial alterations occur because of an additive and progressive cellular necrosis initiated and perpetuated by alterations in the myocardial microcirculation.

At the same time, there could be a consistent autonomic imbalance in the sympathetic and parasympathetic dysfunction in chagasic patients that would predispose their coronary arteries to a constrictor stimulus. The clinical importance of these findings would lie in linking them with the symptoms of angina suffered by chagasic patients and their contribution towards the advance of chronic chagasic cardiomyopathy, and with the patients that have this abnormality of vasomotion in the chronic phase without evidence of ex indeterminate pathology.

Celermajer and team used the test for ischemia by means of brachial ultrasound as a diagnostic method of the endothelial lesion in diverse illnesses like atherosclerosis, in factors of cardiovascular risk ad in other processes in which the endothelial function was disturbed, with excellent sensitivity, specificity and predictive value. Immune alterations are produced in Chagas disease that could play a role in its beginning and evolution. The antibodies could alter the ionic flow of the cardiac cell membranes with irreversible lesions $[15,16$, 18-21].

The management of Chagas disease today in both the diagnosis and treatment is extrapolated from the management of the cardiac pathologies of non-chagasic origins.

Many concerns have arisen concerning the function of new diagnostic tools to evaluate cardiac problems.

\section{RISK SCORE}

Recently our group of investigators established a comprehensive risk score for chronic patients without demonstrable ex indeterminate pathology to develop complications (Mitelman-Gimenez) (Table 1).

The present score (of clinical outcome) was not used

Table 1. Risk score.

\begin{tabular}{|c|c|}
\hline \multicolumn{2}{|l|}{ Exploration of the autonomic nervous system: } \\
\hline Antimuscarinic receptor antibodies & 3 \\
\hline Ergometry & 3 \\
\hline Variability of cardiac frequency & 2 \\
\hline QT dispersion analysis & 2 \\
\hline \multicolumn{2}{|l|}{ Exploration of the endothelium: } \\
\hline Soluble thrombomodulin or the von Willebrand factor & 3 \\
\hline Brachial echo Doppler & 3 \\
\hline \multicolumn{2}{|l|}{ Evaluation of the autonomic myocardial substrate: } \\
\hline Two-dimensional echocardiogram-tissue Doppler Imaging & 4 \\
\hline \multirow[t]{2}{*}{ Signal averaged electrocardiogram } & 3 \\
\hline & $\begin{array}{l}\text { Mild } \\
1-9\end{array}$ \\
\hline \multirow[t]{2}{*}{ Risk score } & $\begin{array}{c}\text { Moderate } \\
10-18\end{array}$ \\
\hline & $\begin{array}{l}\text { Severe } \\
19-23\end{array}$ \\
\hline
\end{tabular}


to determine sudden death but to evaluate a set of risk factors of activity and associated progression with new tools that would permit the detection of critical patients that could develop in their evolution cardiomyopathy, thus, enabling the utilization of new therapeutic strategies. There is a growing consensus that the presence of Trypanosoma cruzi is a necessary condition and sufficient for the development of lesions characterized in chronic Chagas disease, both in animals and humans; and that the eradication of the parasite is a prerequisite in order to avoid the evolution of terminal forms of the disease [22-29].

\section{TREATMENT}

The drugs available today for the etiological treatment of Chagas disease have serious deficiencies particularly due to their very low efficacy in the treatment of chronic infections and their frequent side effects.

The physiopathological mechanisms that trigger the progression of the disease after parasitic aggression could be used in the future to guide the therapeutic process of those patients towards the use of $\beta$-receptor antagonists for the modulation of the coronary vasomotor tone, the angiotensin-converting-enzyme inhibitors to inhibit the vasoconstrictor effect of the angiotensin and the calcium channel blockers to prevent tissue hypoxia because of their vasodilatory and antiplatelet effects.

The use of angiotensin-converting-enzyme (ACE) inhibitors and statins in chronic patients without evidence of ex indeterminate pathology is based on what was presented earlier and will surely open the doors to the application of new therapeutic treatments combined with antiparasitic and those that would act temporarily on the consequences triggered by the action of the Trypanosoma cruzi avoiding the development of cardiomyopathy and its effects.

Recent studies have shown that the statins would improve the endothelial lesion by means of antioxidant and anti-inflammatory actions to increase the activity and expression of the endothelial sintetase nitric oxide that would mean an improvement in the vascular function. Possibly, its beneficial effect in this pathology should be sought in the effects of the immunomodulators. The angiotensin-converting-enzyme inhibitors have also shown in diverse studies the attenuation of the endothelial lesion [30].

Once the lesion is established in the microcirculation, the ischemia would produce myocytolysis, replacing the cardiac tissue for fibrosis.

Chronic chagasic myocarditis may have a process of fibrosis and necrosis as a histologic substrate (disseminated multifocal panmyocarditis characterized by Rosenbaum) that would have a place in an infinite number of microscopic focal points that are at times confluent.
These abnormalities that could compromise the specialized conduction tissue, the common myocardium and the autonomic nervous system could lead to the multiple electrophysiological anomalies. The prevalence and complexity of ventricular arrhythmias are directly related with the extension of the damage. The ventricular extrasystoles and interventricular conduction disturbances would be the earliest ventricular compromise [31].

A close relationship could exist between the severity of the ventricular dysfunction, the ventricular arrhythmias and the probability of sudden death. The angiotensin II receptor antagonist, Losartan inhibits the growth and proliferation of the cardiac muscular cells and the smooth vascular and endothelial cells, inducing the fibroblast apoptosis and activating diverse collagenases. These antiproliferative effects that are associated with the inhibition of the kinase pathway activated by mitogens diminish the cardiovascular fibrosis and the cardiac remodeling as well as the neointima formation in response to the vascular lesion.

Different authors have reported the presence in approximately $30 \%$ of population with serological positive muscarinic antibodies that in many occasions are correlated with the presence of alterations in the variability of frequency and the QT dispersion. This would permit the diagnosing of cardiac dysautonomy and in addition, other authors point out the evolutionary aggravation in those where it is detected. These results would gain importance in the search for therapeutic neutralizers or blockers as well as the removal by means of an immunosorbent, which is done in idiopathic dilated cardiomyopathy [19-22].

\section{CONCLUSION}

We believe that the persistence of the parasite in the infected organism would not be the only mechanism that would have a determinate role in the development of the pathogenesis of the illness and consequently even a successful etiological treatment would not be able to avoid the development of the terminal lesions characterized by the disease. Therefore, the treatment should include the objective to protect the myocardium the statins, angiotensin-converting-enzyme inhibitors and losartan in accordance with the stage of the disease. Chagasic cardiomyopathy would constitute a model of nonischemic vascular cardiomyopathy of parasitic origin that would require the combined action of, immunomodulators, antiinflammatory and parasitic drugs attenuating the endothelial and antifibrotic lesion depending upon the stage of the disease.

\section{REFERENCES}

[1] Schofield, C.J. (1989) American trypanosomiasis. In 
Geographical distribution of arthropod-borne diseases and their principal vectors. World Health Organization manual, WHO/VBC/89.967, 81-92.

[2] Report if a WHO (World Health Organization) informal consultation (jointly organized by WHO headquarters and the WHO regional office for Europe) (2009) Control and prevention of Chagas disease in Europe. Geneva, 1-74.

[3] Kirchhoff, L. (1993) American trypanosomiasis (Chagas' disease): A tropical disease now in the United States. New England Journal of Medicine, 329, 639-644. http://dx.doi.org/10.1056/NEJM199308263290909

[4] Pung, O.J., Banks, C.W., Jones, D.N. and Krissinger, M.W. (1995) Trypanosoma cruzi in wild raccoons, and triatomine bugs in southeast Georgia, USA. Journal of Parasitology, 81, 324-326. http://dx.doi.org/10.2307/3283947

[5] Schofield, C.J. and Galvão, C. (2009) Classification, evolution and species groups within the Triatominae. Acta Tropica, 110, 88-100. http://dx.doi.org/10.1016/j.actatropica.2009.01.010

[6] Morris, S., Tanowitz, H. and Murray, W. (1990) Pathophyphysiological Insights into the cardiomyopathy of Chagas' disease. Circulation, 82, 1900-1908. http://dx.doi.org/10.1161/01.CIR.82.6.1900

[7] Rosemberg, I. and Pereira, M. (1987) Smilitary of cruzan an inhibitor of Trypanosoma cruzi neuraminidase, to high-density lipoprotein. Science, 84, 10-12.

[8] Tarleton, R. (2001) Parasite persistence in the etiology of Chagas disease. International Journal for Parasitology, 31, 549-553. http://dx.doi.org/10.1016/S0020-7519(01)00158-8

[9] Libby, P., Alroy, J. and Pereyra, M.E. (1986) A neuralminidase from Trypanosoma cruzi removes sialic acid from the surface of mammalian myocardial and endothelial cells. Journal of Clinical Investigation, 77, 127-135. http://dx.doi.org/10.1172/JCI112266

[10] Rossi, M.A. and Mengel, J.O. (1992) The pathogenesis of chronic Chagas myocarditis: The role of autoimmune and microvascular factors. Revista do Instituto de Medicina Tropical de São Paulo, 34, 539-599.

[11] Mordini, O., et al. (2010) Council of heart disease and Chagas disease. Time to update the clinical classification of the Chagas disease. Four seasons hotel Buenos aires 20/3/10, Pág, WEB, SIAC.

[12] Mitelman, J., et al. (2011) Consensus of Chagas Mazza Disease. Revista Argentina de Cardiología, 79, 544-564.

[13] Marin-Neto, J.A. (1998) Cardiac dysautonomy and pathogenesis of Chagas' heart disease. International Journal of Cardiology, 66, 129-131.

[14] Redruello, M., Masoli, O., Perez Baliño, N. and team (2003) Endothelial dysfunction in Chronic Chagas disease. Assesment with 99 mTc sestamibi Spect cold pressor test and intracoronary aceltylcholine. Journal of $\mathrm{Nu}$ clear Cardiology, 10, 9-26.

[15] Celermajer, D.S., Sorensen, K.E., Bull, C. and team. (1994) Endothelium dependent dilation in the systemic arteries of asymptomatic subjects relates to coronary risk factors and their interaction. Journal of the American
College of Cardiology, 24, 1468-1474. http://dx.doi.org/10.1016/0735-1097(94)90141-4

[16] Celermajer, D.S., Sorensen, K.E., Geogakopoulos, D. and team (1993) Cigarette smoking is associated with doserelated and potentially reversible impairment of endothelium-dependent dilation. Circulation, 88, 2149-2155. http://dx.doi.org/10.1161/01.CIR.88.5.2149

[17] Van Mourik, J.A., Boertjes, R., Huisveld, I.A. and team (1999) Von Willebrand, factor propeptide in vascular disorders: A tool to distinguish between acute and chronic endothelial cell perturbation. Blood, 94, 179-185.

[18] Iosa, D., Dequatro, V., De-Ping-Lee, D. and team (1990) Pathogenesis of cardiac neuropathy in Chagas disease and the role of autonomic nervous system. Journal of the Autonomic Nervous System, 30, 583-588. http://dx.doi.org/10.1016/0165-1838(90)90107-T

[19] Goin, J.C., Perez Leiros, C., Borda, E. and Sterin-Borda, L. (1997) Interaction of human chagasic IgG with the second extracellular loop of the human heart muscarinic acetylcholine receptor: Functional and pathological implications. The FASEB Journal, 11, 77-83.

[20] Sterin Borda, L. and Borda, E. (2000) Role of neurotransmitter autoantibodies in the pathogenesis of chagasic peripheral dysautonomia. Annals of the New York Academy of Sciences, 917, 273-280. http://dx.doi.org/10.1111/j.1749-6632.2000.tb05393.x

[21] Matsumato, S., Labowsky, V., et al. (2006) Retinal dysfunction in patients with chronic Chagas disease is associated to anti-trypanosoma cruzi antibodies that crossreact with rhodopsim. The Faseb Journal, 20, 550-552.

[22] Salles, G., Xavier, S., Sousa, A., Hasslocher-Moreno, A. and Cardoso, C. (2003) Prognostic value of QT interval parameters for mortality risk stratification in Chagas' disease: Results of a long-term follow-up study. Journal of the American Heart Association, 108, 305-312.

[23] Mitelman, J., Gimenez, L., Diaz, A., et al. (2001) Association between endothelial dysfunction assessed by ischemic test with brachial echo-Doppler and Von Willebrand factor in Chagas disease. Revista Argentina de Cardiología, 69, 275-278.

[24] Gimenez, L., Mitelman, J. and Gonzalez, C. (2003) Antiautonomic receptor antibodies, heart rate variability and arrhythmias in patients with Chagas disease. Revista Argentina de Cardiología, 71, 109-113.

[25] Mitelman, J., Gimenez, L., et al. (2000) Non invasive diagnostic methods for detection of functional anomalies in chronic Chagas disease. Revista Argentina de Cardiología, 68, 797-802.

[26] Mitelman, J., Gimenez, L. and Gonzalez, C. (2013) Valorar la asociación de pruebas diagnósticas incruentas para la detección de alteraciones funcionales miocárdicas autonómicas y endoteliales en la enfermedad de chagas. Revista Guatemalteca de Cardiología, 23, 16-20.

[27] Breithardt, G. and Cain, M. (1991) Flowers standards for analysis of ventricular late potential using high-resolution or signal averaged electrocardiography: A statement by a task force committee of the European Society of Cardiology, the American Heart Association and the American College of Cardiology. Journal of American College of 
Cardiology, 17, 999-1006.

http://dx.doi.org/10.1016/0735-1097(91)90822-Q

[28] Marek, M. (1996) Heart rate variability. Standards of measurement, physiological interpretation and clinical use task force of the European Society of Cardiology and the North American Society of Pacing and Electrophysiology. Circulation, 93, 1043-1065.

[29] Malik, M. and Camm, A.J. (1997) Mystery of QTc interval dispersion. American Journal of Cardiology, 49, 785-
787.

[30] Mitelman, J., Gimenez, L., Ruggiero, M., et al. (2008) Cardiac rehabilitation in chagasic patients with heart failure. Revista Costarricense de Cardiología, 10.

[31] Mitelman, J., Giménez, L., Palacios, K., Tomasella, M., Acuña, L. and Avayú, D. (2011) New paradigms in Chagas Mazza disease.

www.intramed.net/contenidover.asp?contenidoID=71783 